\title{
Ensaios clínicos toxicológicos, fase I, de um fitoterápico composto (Schinus terebinthifolius Raddi, Plectranthus amboinicus Lour e Eucalyptus globulus Labill)
}

\author{
Patrícia Trindade C. Paulo*, Margareth de F. F. M. Diniz, Isac A. de Medeiros, Liana C. S. L. \\ de Morais, Fábia Barbosa de Andrade, Hosana Bandeira Santos
}

\author{
Laboratório de Tecnologia Farmacêutica "Delby Fernandes de Medeiros", Universidade Federal da Paraíba, \\ Caixa Postal 5009, 58051-900 João Pessoa-PB, Brasil
}

\begin{abstract}
RESUMO: Neste estudo, foram realizados ensaios clínicos toxicológicos, fase I, do produto fitoterápico composto pelas plantas medicinais Schinus terebinthifolius Raddi, Plectranthus amboinicus Lour e Eucalyptus globulus Labill. O estudo foi realizado no Hospital Universitário Lauro Wanderley/UFPB/PB e, para isto, foram selecionados 28 voluntários sadios, sendo 14 homens e 14 mulheres que ingeriram por via oral, ininterruptamente durante 8 semanas, $15 \mathrm{~mL}$ do produto, três vezes ao dia; e no $3^{\circ}$ e $7^{\circ}$ dia, $3^{\mathrm{a}}$ e $6^{\mathrm{a}}$ semanas e $24 \mathrm{~h}$ após a $8^{\mathrm{a}}$ semana, foram feitas avaliações clínicas e laboratoriais para análise da toxicidade aguda e crônica. Os resultados obtidos demonstraram que os pacientes não apresentaram alterações clínicas, laboratoriais e reações adversas significantes, apenas pequenas alterações foram detectadas no sangue através da aspartato transaminase (AST) e fosfatase alcalina no grupo feminino para um $p<0,05$; no entanto, estes valores determinados permaneceram dentro dos valores de normalidade para indivíduos adultos. Conclui-se que estes dados, em complementação àqueles obtidos com os estudos pré-clínicos, confirmam a baixa toxicidade do produto fitoterápico.
\end{abstract}

Unitermos: Schinus terebinthifolius, Anacardiaceae, Plectranthus amboinicus, Lamiaceae, Eucalyptus globulus, Myrtaceae, ensaio clínico toxicológico, fase I, fitoterápico composto.

\begin{abstract}
Phase I clinical toxicological assays of a complex herbal medicine (Schinus terebinthifolius Raddi, Plectranthus amboinicus Lour and Eucaliptus globulus Labill)". In this study, phase I clinical toxicological assays of the herbal medicine composed of the medicinal plants Schinus terebinthifolius Raddi, Plectranthus amboinicus Lour and Eucaliptus globulus Labill were performed. The study was carried out at Hospital Universitário Lauro Wanderley/UFPB/PB/Brazil and for this purpose, 28 healthy volunteers were chosen, 14 men and 14 women who ingested $15 \mathrm{~mL}$ of the medicine per oral, with no interruption, three times a day; and on the $3^{\text {rd }}$ and $7^{\text {th }}$ days, on the $3^{\text {rd }}$ and $6^{\text {th }}$ weeks and $24 \mathrm{~h}$ after the $8^{\text {th }}$ week, clinical and laboratory evaluations were performed to analyze the acute and chronic toxicity. As results, the patients did not show significant clinical and laboratory alterations and adverse reactions, only little alterations were detected in blood through aspartate transaminase (AST) and alkaline phosphatase in the female group to a $\mathrm{p}<$ 0.05; however, these values are according to the normality standard for adult individuals. It can be concluded that these data, complementary to those obtained with the preclinical studies, confirm the low toxicity of the herbal medicine.
\end{abstract}

Keywords: Schinus terebinthifolius, Anacardiaceae, Plectranthus amboinicus, Lamiaceae, Eucalyptus globulus, Myrtaceae, clinical toxicological assay, phase I, complex herbal medicine.

\section{INTRODUÇÃO}

O produto fitoterápico composto pelas plantas medicinais Schinus terebinthifolius Raddi, Plectranthus amboinicus Lour e Eucalyptus globulus Labill, é um medicamento tradicional com uso consolidado em toda a Paraíba, além dos estados do Rio Grande do Norte, Pernambuco e Ceará (Brasil). Vem sendo usado há mais de 100 anos para várias afecções como aftas, amigdalites, faringites, picadas de insetos, furúnculos, úlceras varicosas, hemorragias nasais, rinites, acne e outros. É também, utilizado usado para tratar acidez, mau hálito, enxaqueca, enjôos e má digestão (Bertoldi et al., 2004).

A partir do ano de 2000, a Agência Nacional de Vigilância Sanitária (Anvisa) com o intuito de regularizar os produtos fitoterápicos existentes no mercado brasileiro, exigiu que fossem feitos nestes produtos os testes pré-clínicos e clínicos, até então só exigidos dos medicamentos alopáticos. Para a obtenção do registro destes produtos na Anvisa, esta editou a Resolução RDC n ${ }^{\circ} 48$ de 16 de março de 2004, que dispõe sobre o registro de medicamentos fitoterápicos, e define 
como medicamento fitoterápico, (medicamento obtido empregando-se exclusivamente matérias-primas ativas vegetais, e a sua eficácia e segurança é validada através de levantamentos etnofarmacológicos de utilização, documentações tecnocientíficas em publicações ou ensaios clínicos fase III). Para atingir esta finalidade, necessita-se anteriormente, realizar ensaios pré-clínicos de toxicidade do produto, regido pela resolução RE nº0/2004 da Anvisa (Brasil, 2004a,b).

Buscando desenvolver estas fases é que o produto fitoterápico de que trata este trabalho vem sendo estudado.

A Schinus terebinthifolius é uma árvore de pequeno a médio porte, pertencente à família Anacardiaceae, com propriedades adstringente, antiséptica e anti-inflamatória na medicina popular e usos em preparações cosméticas (Biavatti et al., 2007; Agra et al., 2007, 2008). É originária da América do Sul, especialmente do Brasil, Paraguai e Argentina e é conhecida popularmente como aroeira da praia, aroeira branca, aroeira vermelha dentre outras (Diniz et al., 1999). O seu extrato contém taninos, terpenos, flavonóides e saponinas, destes componentes, as propriedades potenciais e antioxidantes foram atribuídas aos flavonóides (Carvalho et al., 2003). Em estudos científicos realizados com a casca do caule foi detectada a presença de taninos, que lhe conferem a ação adstringente, desinfetante e antiinflamatório. Em estudos pré-clínicos, utilizando o decocto, ficou comprovado a sua eficácia como protetor gástrico, elevando o $\mathrm{pH}$ do suco gástrico de 5,85 para 6,57 (Santos et al., 2006). Foi comprovado também, em estudos clínicos a ação terapêutica em cervicites e cérvico-vaginites crônicas, utilizando tampões intravaginais, em contato com a cérvix durante 24 horas (Lima et al., 2006). Ensaios de toxicidade foram realizados e detectados que a resina produzida por esta planta, em contato com a pele causa dermatite alérgica (Moraes et al., 2004). O hidrolato de um preparado medicamentoso, composto por três plantas, entre elas, o $S$. terebinthifolius, mostrou propriedades antiinflamatórias (Medeiros et al., 2007).

A espécie Plectranthus amboinicus Lour pertence à família das Lamiaceae. É uma planta aromática nativa da Ásia Oriental, encontrada em toda a América Tropical, desde as Antilhas até o Sul do Brasil (Lukhoka et al., 2006). O timol e o carvacrol presentes no óleo essencial apresentam uma atividade antibacteriana e devido a isso, ocorre uma melhora nas patologias do trato respiratório do homem. O carvacrol tem atividade germicida, antisséptica e antifúngica (Matos, 2000; Oliveira et al., 2006; Oliveira et al., 2007). De acordo com estudos sobre a toxicidade desta espécie, foi citado apenas, que quando utilizada antes de dormir pode provocar insônia, bem como algumas contra-indicações como a ingestão em grandes quantidades por crianças e lactantes (Minker et al., 2007).
A espécie Eucalyptus globulus Labill pertence à família Myrtaceae (Cronquist, 1981). O gênero Eucalyptus possue cerca de 400 espécies, que constituem este grande e inconfundível gênero dentro desta família. É bastante utilizado no reflorestamento e na produção de celulose (Yang \& Guo, 2007). Estudos farmacológicos demonstraram que a principal atividade do Eucalyptus globulus, concentra-se ao nível do aparelho respiratório em função de seu óleo essencial. O mesmo tem sido usado na medicina tradicional no tratamento de bronquite, asma e de outras doenças respiratórias (Vigo et al., 2004; Tavares et al., 2006). Estudos in vitro, têm demonstrado atividade antiviral frente à Influenza $A$. Sobre a toxicidade, o Eucaliptus globulus apresentou, nas doses recomendadas, uma boa tolerabilidade, mas, com doses altas poderá ocasionar náuseas, vômitos, gastroenterites, hematúria, e neurotoxidade. Aplicações tópicas têm provocado reações alérgicas locais, em alguns casos, tais como urticária e eczema (Liu et al.,2003). E não deve ser administrado junto a sedativo, analgésico e anestésico, por perigo de potencializações das respectivas ações (Canigueral \& Vila 1998). A grande utilização do Eucalyptus globulus levou à sua inclusão na primeira, segunda e quarta edição da Farmacopéia Brasileira (Brandão et al., 2006) e, é uma das plantas com registro na Anvisa (Carvalho et al., 2008).

$\mathrm{O}$ produto fitoterápico composto pelas três plantas acima descritas passou também por testes préclínicos. Avaliou-se a toxicidade em animais de laboratório (ratos e cães), de acordo com a portaria 116/96, vigente na época, da Secretaria de Vigilância Sanitária, atual Anvisa (Brasil, 1996a). Os resultados obtidos demonstraram que na investigação da toxicidade aguda e crônica do produto em cães sem raça definida (SRD), não houve mortes e nem alterações significantes no comportamento e nem nos dados laboratoriais; nos ratos (Rattus novergicus) não houve mortes e nem alterações comportamentais, apenas demonstraram um discreto aumento da Alanina Transaminase (ALT) e Aspartato Transaminase (AST) no sangue, mas foram reversíveis. Concluiu-se neste estudo, que este produto é de baixa toxicidade, porque usou três doses, na qual uma delas foi semelhante à usada em seres vivos, e cinco vezes esta dose até 25 vezes e não houve mortes e nem alterações significantes nos animais, viabilizando assim, os ensaios clínicos (Toscano, 2004).

Com isto, foram realizados ensaios clínicos toxicológicos referentes à fase I em seres humanos, deste produto fitoterápico composto, para favorecer o registro ou não deste na Anvisa de acordo com a RDC nº 48/2004 (Brasil, 2004a).

\section{MATERIAL E MÉTODOS}

\section{Produto utilizado}

É um fitoterápico composto por Schinus terebinthifolius Raddi, Plectranthus amboinicus Lour e Eucalyptus globulus Labill, tratando-se de um hidrolato já comercializado e largamente utilizado há mais de 100 
anos no Nordeste do Brasil. Amostras foram cedidas pela própria empresa que o produz, e foram utilizados 90 frascos de $500 \mathrm{ml}$, pertencente ao lote $\mathrm{n}^{\circ} 1135$ de validade (12/2007). Para a produção do medicamento foram utilizadas folhas verdes de aroeira da praia (Schinus terebinthifolius Raddi), de hortelã da folha grossa (Plectranthus amboinicus Lour), ambas cultivadas no horto da própria sede da empresa. Foi usada também a essência do eucalipto (extrato do óleo essencial de Eucalyptus globulus Labill). Amostras de aroeira e de hortelã da folha grossa foram identificadas pela Curadora Marlene Barbosa do Herbário do Centro de Ciências Biológicas da Universidade Federal de Pernambuco.

\section{Local da pesquisa}

Este estudo foirealizado no Hospital Universitário Lauro Wanderley (HULW) da Universidade Federal da Paraíba (UFPB), onde os exames complementares foram realizados no laboratório de análises clínicas e na sala de cardiologia daquele nosocômio e os estudos complementares foram feitos no Laboratório de Tecnologia Farmacêutica Delby Fernandes de Medeiros da UFPB. Este foi aprovado pelo o Comitê de Ética e Pesquisa do HULW sob o parecer de $n^{\circ} 136$.

\section{Voluntários}

Foi feito um estudo clínico de fase I, de acordo com as Resoluções $\mathrm{n}^{\circ} 196 / 96$ (Brasil, 1996b) e 251/97 (Brasil, 1997) do CNS, do tipo randomizado com a participação voluntária de indivíduos, após assinarem o Termo de Consentimento livre e esclarecido. Foram utilizados 28 seres humanos, 14 homens e 14 mulheres entre 20 e 40 anos de idade, previamente selecionados, identificando ser devidamente sadios para poderem participar desta pesquisa, tendo sido feita uma avaliação clínica e laboratorial completa.

\section{Aparelhagem}

Foi utilizada uma Centrífuga (BIO ENG ${ }^{\circledR}$ BE 4000) para a separação do soro e da urina. As determinações bioquímicas no soro humano foram feitas através de analisador bioquímico automático. As determinações dos íons (sódio, potássio e cálcio) foram realizadas através do analisador de íons seletivos. Para a obtenção dos hemogramas e contagem de plaquetas foi utilizado o analisador hematológico celular automático. $\mathrm{O}$ microscópio (RML5® Askmania-Germany) foi utilizado para a análise dos sedimentos urinários, e fitas reagentes (Multistix) foram utilizadas para uroanálises. Também foi utilizado um eletrocardióografo (ECAFIX) de 12 derivações para captação e amplificação de diferenças de potenciais cardíacos, entre os vários pontos de colocação de eletrodos no corpo do paciente.

\section{Protocolo experimental}

Os voluntários tomaram uma dose de $15 \mathrm{~mL}$ do produto fitoterápico por via oral, 3 vezes ao dia durante 8 semanas, e foi doado um copo-medida para tal administração. A partir do $1^{\circ}$ dia de ingestão até o $7^{\circ}$ dia avaliou-se a toxicidade aguda e depois até a $8^{a}$ semana, avaliou-se a toxicidade crônica. No $3^{\circ}$ e $7^{\circ}$ dia, na $3^{\mathrm{a}}$ e $6^{\mathrm{a}}$ semana e 24 horas após a $8^{\mathrm{a}}$ semana de ingestão do produto, os indivíduos (homens e mulheres) vieram ao hospital e foram submetidos a uma avaliação clínica e laboratorial do seu estado geral. Eles realizaram os seguintes exames: glicemia, creatinina fosfoquinase (CPK), triglicerídios, colesterol total e frações, lactato desidrogenase (LDH), amilase pancreática, sódio, potássio, aspartato transaminase, alanina transaminase, bilirrubina total e frações, gama GT, fosfatase alcalina, proteína total e frações, creatinina, ácido úrico, uréia, hemograma completo, contagem de plaquetas e exame de urina tipo I. Foi realizado também um eletrocardiograma de doze derivações nos mesmos.

Durante todo o curso da pesquisa, os voluntários foram instruídos a comunicarem aos pesquisadores, qualquer sinal ou sintoma que porventura apresentassem de reação adversa e também receberam um questionário para preencher sobre isto, se fosse o caso.

\section{Critérios de exclusão}

Foram excluídos do estudo, os indivíduos que apresentaram nos exames iniciais alterações clínicas e/ou laboratoriais; que revelassem alguma disfunção hepática, renal, cardíaca; pacientes grávidas, e que faziam uso de álcool e/ou de alguma medicação.

\section{Análises estatísticas}

A avaliação dos parâmetros bioquímicos e hematológicos dos voluntários foi realizada comparandose os resultados obtidos ao longo do tratamento com aqueles da seleção inicial de cada voluntário (tempo basal), no qual estes se encontravam na faixa de normalidade de um indivíduo considerado sadio. Os valores foram expressos em média \pm erro padrão da média (e.p.m.) dos 28 voluntários, separando-os por sexos, de acordo com o tipo de exame e o período de avaliação. Foi utilizada a Análise de Variância ANOVA de uma via, seguido pelo teste de Bonferroni a posteriori quando necessário. Todos os dados foram analisados pelo o programa estatístico Graph Pad Prism ${ }^{\circledR}$ versão 3.03.

\section{RESULTADOS}

Foram inicialmente selecionados 19 mulheres e 20 homens, mas de acordo com o padrão de normalidade que não alcançaram, foram eliminadas 5 mulheres e 6 homens, restando ao final, 14 mulheres e 14 homens 
necessários a realização da pesquisa. O item adesão foi atingido na sua integridade, no qual todos cumpriram integralmente as 8 (oito)semanas e os resultados obtidos demonstraremos a seguir. Iniciaremos pelos os exames hematológicos dos indivíduos (homens e mulheres), que durante este período não apresentaram alterações significativas de acordo com o teste estatístico utilizado, (Tabela 1 e 2).

Os resultados obtidos nos exames bioquímicos dos voluntários masculinos não demonstraram alterações significantes de acordo com o teste estatístico, em relação aos valores normais destes parâmetros obtidos inicialmente (Tabela 3 e 4).

Após a ingestão do produto fitoterápico, os resultados obtidos nos exames das voluntárias femininas, não demonstraram alterações em relação aos valores normais obtidos inicialmente, apenas o aspartato transaminase e a fosfatase alcalina demonstraram

Tabela 1. Média e desvio padrão dos valores hematológicos obtidos nos homens.

\begin{tabular}{c|c|c|c|c|c|c}
\hline \multirow{2}{*}{ Tempo } & BASAL & $3^{\circ}$ DIA & $7^{\circ}$ DIA & $3^{\circ}$ SEMANA & $6^{\circ}$ SEMANA & 24h/8 $8^{\circ}$ SEMANA \\
\cline { 2 - 6 } & X E.P. & X \pm E.P. & X \pm E.P. & X \pm E.P. & X \pm E.P. & X \pm E.P. \\
\hline $\begin{array}{c}\text { Hemácias } \\
4,5 \text { a } 5,5 \text { milh./mm }\end{array}$ & $5,1 \pm 0,07$ & $5,1 \pm 0,09$ & $5,0 \pm 0,1$ & $5,1 \pm 0,1$ & $5,2 \pm 0,07$ & $5,0 \pm 0,1$ \\
\hline $\begin{array}{c}\text { Hemoglobina } \\
13,5 \text { a } 18 \mathrm{~g} / \mathrm{dl}\end{array}$ & $15,6 \pm 0,2$ & $15,4 \pm 0,3$ & $15,6 \pm 0,2$ & $15,4 \pm 0,2$ & $15,4 \pm 0,3$ & $15,0 \pm 0,3$ \\
\hline $\begin{array}{c}\text { Hematócrito } \\
40 \text { a } 52 \%\end{array}$ & $45 \pm 0,5$ & $44 \pm 0,7$ & $45 \pm 0,8$ & 450,9 & $45 \pm 0,8$ & $44 \pm 0,8$ \\
\hline $\begin{array}{c}\text { Leucócitos } \\
5.000 \text { a } 10.000 \mathrm{~mm}^{3}\end{array}$ & $7.307 \pm 501,8$ & $6.872 \pm 490$ & $6.853 \pm 408$ & $7.269 \pm 507$ & $7.038 \pm 432$ & $6.948 \pm 544$ \\
\hline $\begin{array}{c}\text { Neutrófilos } \\
2.500 \text { a } 6.500 \mathrm{~mm}^{3}\end{array}$ & $4.213 \pm 471$ & $3.905 \pm 427$ & $3.698 \pm 316$ & $4.178 \pm 499$ & $3.993 \pm 441$ & $3.777 \pm 534$ \\
\hline $\begin{array}{c}\text { Linfócitos } \\
1.000 \text { a } 3.500 \mathrm{~mm}^{3}\end{array}$ & $2.341 \pm 128$ & $2.248 \pm 133$ & $2.276 \pm 142$ & $2.279 \pm 182$ & $2.370 \pm 169$ & $2.400 \pm 152$ \\
\hline $\begin{array}{c}\text { Monócitos } \\
100 \text { a } 800 \mathrm{~mm}^{3}\end{array}$ & $400 \pm 43,6$ & $370 \pm 51,9$ & $441 \pm 74,3$ & $436 \pm 51,7$ & $379 \pm 63,5$ & $327 \pm 55,8$ \\
\hline $\begin{array}{c}\text { Plaquetas } \\
150.000 \text { a } 400.000 \mathrm{~mm}^{3}\end{array}$ & $268 \pm 18,1$ & $280 \pm 16,7$ & $265 \pm 16,6$ & $284 \pm 17$ & $284 \pm 17,8$ & $281 \pm 17,2$ \\
\hline \begin{tabular}{c} 
ANOVA de \\
\hline
\end{tabular} & & & & & \\
\hline
\end{tabular}

ANOVA de uma via / Teste de Bonferroni (Média $\pm \mathrm{EP}, \mathrm{n}=14$ homens ).

Tabela 2. Média e desvio padrão dos valores hematológicos obtidos nas mulheres.

\begin{tabular}{|c|c|c|c|c|c|c|}
\hline Tempo & BASAL & $3^{\circ}$ DIA & $7^{\circ}$ DIA & $3^{\circ}$ SEMANA & $6^{\circ}$ SEMANA & $24 \mathrm{~h} / 8^{\circ} \mathrm{SEMANA}$ \\
\hline Exame & $\mathrm{X} \pm$ E.P. & $\mathrm{X} \pm$ E.P. & $\mathrm{X} \pm$ E.P. & $\mathrm{X} \pm$ E.P. & $\mathrm{X} \pm$ E.P. & $\mathrm{X} \pm$ E.P. \\
\hline $\begin{array}{c}\text { Hemácias } \\
4,5 \text { a } 5,5 \text { milh. } / \mathrm{mm}^{3}\end{array}$ & $4,5 \pm 0,06$ & $4,5 \pm 0,05$ & $4,5 \pm 0,05$ & $4,5 \pm 0,05$ & $4,5 \pm 0,06$ & $4,5 \pm 0,06$ \\
\hline $\begin{array}{r}\text { Hemoglobina } \\
13,5 \text { a } 18 \mathrm{~g} / \mathrm{dl} \\
\end{array}$ & $13,2 \pm 0,2$ & $13,1 \pm 0,2$ & $12,9 \pm 0,2$ & $13,0 \pm 0,1$ & $13,1 \pm 0,2$ & $13,1 \pm 0,2$ \\
\hline $\begin{array}{c}\text { Hematócrito } \\
40 \text { a } 52 \%\end{array}$ & $39 \pm 0,5$ & $38 \pm 0,6$ & $39 \pm 0,5$ & $38 \pm 0,4$ & $39 \pm 0,5$ & $38 \pm 0,5$ \\
\hline $\begin{array}{c}\text { Leucócitos } \\
5.000 \mathrm{a} 10.000 \mathrm{~mm}^{3}\end{array}$ & $7.844 \pm 575$ & $7.151 \pm 581$ & $6.922 \pm 570$ & $6.849 \pm 524$ & $7.310 \pm 523$ & $6.836 \pm 533$ \\
\hline $\begin{array}{c}\text { Neutrófilos } \\
2.500 \text { a } 6.500 \mathrm{~mm}^{3}\end{array}$ & $4.931 \pm 492$ & $4.204 \pm 476$ & $4.209 \pm 472$ & $4.058 \pm 439$ & $4.382 \pm 345$ & $4.025 \pm 375$ \\
\hline $\begin{array}{c}\text { Linfócitos } \\
1.000 \text { a } 3.500 \mathrm{~mm}^{3}\end{array}$ & $2.362 \pm 176$ & $2.193 \pm 145$ & $2.062 \pm 161$ & $2.061 \pm 128$ & $2.340 \pm 172$ & $2.242 \pm 172$ \\
\hline $\begin{array}{c}\text { Monócitos } \\
100{\mathrm{a} 800 \mathrm{~mm}^{3}}^{3}\end{array}$ & $376 \pm 38$ & $451 \pm 58$ & $384 \pm 49$ & $414 \pm 40$ & $342 \pm 45$ & $343 \pm 47$ \\
\hline $\begin{array}{c}\text { Plaquetas } \\
150.000 \mathrm{a} 400.000 \mathrm{~mm}^{3}\end{array}$ & $265 \pm 18,2$ & $264 \pm 14,8$ & $257 \pm 13,4$ & $252 \pm 11,8$ & $279 \pm 16,1$ & $260 \pm 12,8$ \\
\hline
\end{tabular}

ANOVA de uma via / Teste Bonferroni (Média \pm EP, $n=14$ mulheres). 
alterações estatisticamente significantes, mas permaneceram dentro do limite de normalidade. (Tabela 5 e 6$)$.

Os resultados obtidos através dos exames de urina realizados, durante todo o transcorrer da pesquisa, não demonstraram alterações significantes em relação aos valores normais obtidos inicialmente. Os exames de eletrocardiogramas demonstraram que a administração do produto fitoterápico experimental, também não provocou alterações estatisticamente significantes nos homens nem nas mulheres. E a partir dos questionários respondidos pelos voluntários, foram identificadas algumas reações adversas, que ocorreram de forma esporádica e não justificaram a interrupção da pesquisa. Os efeitos adversos mais comuns estão descritos na Tabela 7.

\section{DISCUSSÃO}

Este estudo foi desenvolvido para investigar a toxicidade clínica, fase I, em seres humanos, do produto fitoterápico composto pelas plantas medicinais Schinus terebinthifolius Raddi, Plectranthus amboinicus Lour, Eucalyptus globulus Labill.

Inicialmente, foram avaliados os parâmetros hematológicos e bioquímicos dos pacientes. Nos hematológicos não foram encontrados nenhuma alteração estatisticamente significante, e nem nos exames bioquímicos dos homens, apenas pequenas alterações significantes no exame aspartato transaminase (AST) e na fosfatase alcalina no grupo das mulheres.

Os valores normais de AST/TGO para as mulheres é de 10 a $31 \mathrm{U} / \mathrm{L}$. Com a ingestão do produto fitoterápico, os resultados obtidos nas voluntárias femininas permaneceram normais até a $6^{\mathrm{a}}$ semana, mas 24 horas após a $8^{\mathrm{a}}$ semana, sofreram alterações significantes, de acordo com o teste ANOVA seguido do teste de Bonferroni. Mas, não foi considerado como importante este resultado, apesar do desvio para a direita (aumento) em relação ao valor basal, porque estes dados obtidos mantiveram-se dentro do limite de normalidade para adultos femininos, corroborando com os dados obtidos por Dufour (2005).

Os valores obtidos da fosfatase alcalina nos resultados das voluntárias femininas, mostraram alterações a partir da $3^{a}$ semana de ingestão do produto, com um desvio para a esquerda (diminuição) em relação ao valor basal, estatisticamente significativo de acordo com os testes utilizados, e estariam de acordo com os estudos

Tabela 3. Média e desvio padrão dos valores bioquímicos obtidos nos homens.

\begin{tabular}{|c|c|c|c|c|c|c|}
\hline Tempo & BASAL & $3^{\circ}$ DIA & $7^{\circ}$ DIA & $3^{\circ}$ SEMANA & $6^{\circ}$ SEMANA & $24 \mathrm{~h} / 8^{\circ}$ SEMANA \\
\hline Exame & $\bar{X} \pm$ E.P. & $\bar{X} \pm$ E.P. & $\bar{X} \pm$ E.P. & $\bar{X} \pm$ E.P. & $\bar{X} \pm$ E.P. & $\bar{X} \pm$ E.P. \\
\hline $\begin{array}{c}\text { Glicemia } \\
(70,0 \text { a } 110,0 \mathrm{mg} / \mathrm{dl}) \\
\end{array}$ & $89,8 \pm 2,5$ & $89,8 \pm 1,9$ & $88,7 \pm 2,1$ & $85,9 \pm 1,7$ & $87,8 \pm 2,5$ & $89,1 \pm 4,9$ \\
\hline $\begin{array}{l}\text { Colesterol total } \\
(\text { Até } 200 \mathrm{mg} / \mathrm{dl})\end{array}$ & $199 \pm 12,5$ & $200 \pm 13,5$ & $201 \pm 13$ & $201 \pm 12,3$ & $194 \pm 9,8$ & $194 \pm 9,8$ \\
\hline $\begin{array}{l}\text { HDL - Colesterol } \\
\text { (Até } 65 \mathrm{mg} / \mathrm{dl})\end{array}$ & $49,1 \pm 4,3$ & $45,9 \pm 2,6$ & $47,1 \pm 2,5$ & $46,1 \pm 2,6$ & $47 \pm 2,5$ & $56,3 \pm 5,2$ \\
\hline $\begin{array}{c}\text { LDL - Colesterol } \\
(\text { Menor que } 130 \mathrm{mg} / \mathrm{dl})\end{array}$ & $127,1 \pm 11,2$ & $132,1 \pm 12,2$ & $131 \pm 11,2$ & $131,4 \pm 10,9$ & $124,1 \pm 8,9$ & $117,2 \pm 6,9$ \\
\hline $\begin{array}{l}\text { VLDL - Colesterol } \\
\text { (Até } 30 \mathrm{mg} / \mathrm{dl})\end{array}$ & $22 \pm 2,4$ & $21,5 \pm 2,5$ & $22,5 \pm 2,9$ & $22,8 \pm 3,4$ & $22,5 \pm 3,2$ & $20,1 \pm 3,6$ \\
\hline $\begin{array}{c}\text { Uréia } \\
(10 \text { a } 50 \mathrm{mg} / \mathrm{dl})\end{array}$ & $26,9 \pm 1,6$ & $28,8 \pm 2,3$ & $29 \pm 2,2$ & $26,6 \pm 1,4$ & $28,3 \pm 1,7$ & $29,6 \pm 2,2$ \\
\hline $\begin{array}{c}\text { Creatinina } \\
(0,4 \text { a } 1,4 \mathrm{mg} / \mathrm{dl})\end{array}$ & $0,6 \pm 0,03$ & $0,7 \pm 0,05$ & $0,7 \pm 0,03$ & $0,7 \pm 0,01$ & $0,6 \pm 0,02$ & $0,6 \pm 0,02$ \\
\hline $\begin{array}{c}\text { CPK } \\
\text { Homem: } \leq 190 \mathrm{U} / 1 \\
\text { Mulher: } \leq 175 \mathrm{U} / 1\end{array}$ & $104,6 \pm 11,8$ & $133,1 \pm 14,5$ & $156 \pm 33$ & $173 \pm 35$ & $136,9 \pm 22,6$ & $168 \pm 53,7$ \\
\hline $\begin{array}{c}\text { Ácido Ürico } \\
\text { Homem: } 3 \text { a } 7 \mathrm{mg} / \mathrm{dl} \\
\text { Mulher: } 2 \text { a } 5 \mathrm{mg} / \mathrm{dl}\end{array}$ & $3,8 \pm 0,2$ & $4,8 \pm 0,3$ & $4,3 \pm 0,3$ & $5,1 \pm 0,3$ & $4,1 \pm 0,3$ & $4,7 \pm 0,2$ \\
\hline $\begin{array}{c}\text { Trigliceridios } \\
\text { (Menor que } 200 \mathrm{mg} / \mathrm{dl} \text { ) }\end{array}$ & $112 \pm 12,2$ & $109,4 \pm 12,5$ & $114,6 \pm 15$ & $116 \pm 17,2$ & $114,1 \pm 16,1$ & $120,3 \pm 24,7$ \\
\hline $\begin{array}{c}\text { Sódio } \\
(132 \mathrm{a} 148 \mathrm{mEq} / \mathrm{l}) \\
\end{array}$ & $141,9 \pm 0,4$ & $141,5 \pm 0,3$ & $140,9 \pm 0,4$ & $141,4 \pm 0,5$ & $141,3 \pm 0,5$ & $140 \pm 0,8$ \\
\hline $\begin{array}{c}\text { Potássio } \\
(3,5 \text { a } 5,5 \mathrm{mEq} / \mathrm{l})\end{array}$ & $4,4 \pm 0,1$ & $4,3 \pm 0,08$ & $4,3 \pm 0,07$ & $4,3 \pm 0,09$ & $4,3 \pm 0,1$ & $4,2 \pm 0,1$ \\
\hline
\end{tabular}

ANOVA de uma via / Teste de Bonferroni (Média \pm EP, $n=14$ homens). 
realizados por Bertolami (2005), visto que o uso contínuo de plantas medicinais provoca alterações hepatopáticas nos seres humanos. Mas, apesar destas alterações terem sido identificadas, estes valores permaneceram dentro do padrão de normalidade para os adultos do sexo feminino.

Comparando-se com os ensaios pré-clínicos realizados nos ratos (Rattus novergicus), houve naquele estudo, um discreto aumento da alanina transaminase (ALT) e aspartato transaminase (AST) no sangue dos animais (Toscano, 2004). Mas, nos seres humanos apenas no rupo das mulheres houve alteração significante, e não consideramos estas alterações como relevantes, para este ensaio toxicológico clínico, porque permaneceram dentro dos valores normais.

Em relação ao exame de urina tipo I, este constitui um recurso laboratorial de largo emprego na clínica, sendo capaz de fornecer valiosos elementos para elucidação diagnóstica (Bottini \& Garlipp 2006). Este exame compreende desde a descrição dos caracteres gerais da urina, o exame químico até o estudo microscópico do sedimento. A análise dos resultados revelou que a administração do produto fitoterápico nas 08 semanas, não provocou alterações significantes nos índices normais dos voluntários obtidos no tempo basal.
A avaliação clínica constituiu-se, também, um aspecto de investigação importante, utilizando os parâmetros de pressão arterial, freqüência de pulso, temperatura e outros. Todos estes aspectos foram avaliados pelo o médico, envolvido na pesquisa, e permaneceram dentro do padrão de normalidade para os indivíduos masculinos e femininos.

Algumas reações adversas foram evidenciadas no decorrer do estudo, como: cefaléia, náusea, azia, dor no estômago, constipação, gosto estranho na boca e diminuição do apetite, mas o número de voluntários acometidos em relação ao número total foi pequeno, e os sintomas relatados estão também descritos por Motta \& Bianchi (2005) e desapareceram até 03 dias após a suspensão do produto. Demonstrando assim, que estas reações registradas são reversíveis e de natureza leve que não necessitam da retirado do produto em estudo.

\section{CONCLUSÃO}

Fundamentados nos dados obtidos neste estudo, conclui-se que a ingestão oral do produto fitoterápico composto pelas plantas medicinais Schinus terebinthifolius Raddi, Plectranthus amboinicus Lour e Eucalyptus

Tabela 4. Média e desvio padrão dos valores bioquímicos obtidos nos homens (continuação).

\begin{tabular}{|c|c|c|c|c|c|c|}
\hline Tempo & BASAL & $3^{\circ}$ DIA & $7^{\circ}$ DIA & $3^{\circ}$ SEMANA & $6^{\circ}$ SEMANA & $24 \mathrm{~h} / 8^{\circ}$ SEMANA \\
\hline Exame & $\bar{X} \pm$ E.P. & $\bar{X} \pm$ E.P. & $\bar{X} \pm$ E.P. & $\bar{X} \pm$ E.P. & $\bar{X} \pm$ E.P. & $\bar{X} \pm$ E.P. \\
\hline $\begin{array}{c}\text { ALT/TGP } \\
\text { Homem:9 a } 43 \mathrm{U} / 1 \\
\text { Mulher: } 9 \text { a } 36 \mathrm{U} / 1\end{array}$ & $31,6 \pm 1,1$ & $27,9 \pm 2,8$ & $30,4 \pm 2,9$ & $35,8 \pm 2,5$ & $28,2 \pm 1,9$ & $35,3 \pm 4,0$ \\
\hline $\begin{array}{c}\text { AST/TGO } \\
\text { Homem:12 - } 46 \mathrm{U} / 1 \\
\text { Mulher: } 10-31 \mathrm{U} / 1\end{array}$ & $27,6 \pm 1,7$ & $24 \pm 1,9$ & $27,3 \pm 2,1$ & $28,3 \pm 2,2$ & $26,8 \pm 1,8$ & $32,7 \pm 4,3$ \\
\hline $\begin{array}{l}\text { Bilirrubina Total } \\
(0,2 \text { a } 1,0 \mathrm{mg} / \mathrm{dl})\end{array}$ & $0,7 \pm 0,05$ & $0,8 \pm 0,08$ & $0,7 \pm 0,07$ & $0,7 \pm 0,07$ & $0,6 \pm 0,06$ & $0,7 \pm 0,06$ \\
\hline $\begin{array}{l}\text { Bilirrubina Direta } \\
(0,05 \text { a } 0,3 \mathrm{mg} / \mathrm{dl})\end{array}$ & $0,1 \pm 0,01$ & $0,2 \pm 0,02$ & $0,1 \pm 0,01$ & $0,1 \pm 0,02$ & $0,1 \pm 0,01$ & $0,2 \pm 0,02$ \\
\hline $\begin{array}{c}\text { Bilirrubina Indireta } \\
\text { (Até } 0,8 \mathrm{mg} / \mathrm{dl} \text { ) }\end{array}$ & $0,6 \pm 0,05$ & $0,6 \pm 0,08$ & $0,6 \pm 0,06$ & $0,6 \pm 0,07$ & $0,5 \pm 0,06$ & $0,6 \pm 0,05$ \\
\hline $\begin{array}{l}\text { Fosfatase Alcalina } \\
\text { (Menor que } 270 \mathrm{U} / 1 \text { ) }\end{array}$ & $70 \pm 5,4$ & $57,9 \pm 3,6$ & $57,9 \pm 3,2$ & $60,8 \pm 3,9$ & $57,7 \pm 3,0$ & $62,8 \pm 5,4$ \\
\hline $\begin{array}{c}\text { Gama GT } \\
\text { Homem:8 a } 61 \mathrm{U} / 1 \\
\text { Mulher: } 5 \text { a } 36 \mathrm{U} / 1\end{array}$ & $32,4 \pm 3,6$ & $30,6 \pm 2,9$ & $29,9 \pm 2,9$ & $30,6 \pm 4,2$ & $28,7 \pm 3,1$ & $27,9 \pm 3,3$ \\
\hline $\begin{array}{r}\text { Proteinas Totais } \\
(6,0 \text { a } 8,5 \mathrm{~g} / \mathrm{dl}) \\
\end{array}$ & $7,4 \pm 0,1$ & $7,7 \pm 0,1$ & $7,7 \pm 0,1$ & $7,6 \pm 0,1$ & $7,5 \pm 0,1$ & $7,5 \pm 0,1$ \\
\hline $\begin{array}{c}\text { Albumina } \\
(3,5 \text { a } 5,5 \mathrm{~g} / \mathrm{dl})\end{array}$ & $4,4 \pm 0,07$ & $4,5 \pm 0,07$ & $4,5 \pm 0,06$ & $4,4 \pm 0,06$ & $4,5 \pm 0,07$ & $4,5 \pm 0,1$ \\
\hline $\begin{array}{c}\text { Globulina } \\
(1,8 \text { a } 3,8 \mathrm{~g} / \mathrm{dl})\end{array}$ & $3,1 \pm 0,2$ & $3,4 \pm 0,2$ & $3,3 \pm 0,2$ & $3,3 \pm 0,2$ & $3,2 \pm, 02$ & $3,2 \pm, 02$ \\
\hline $\begin{array}{c}\text { Amilase Pancreática } \\
(25 \mathrm{a} 125 \mathrm{U} / \mathrm{l})\end{array}$ & $85,6 \pm 9,5$ & $92,3 \pm 8,5$ & $89,3 \pm 8,3$ & $97,8 \pm 11,7$ & $83,9 \pm 7,3$ & $77,5 \pm 8,5$ \\
\hline $\begin{array}{c}\text { LDH (Lactato Desidrogrnase } \\
\text { (210 a } 425 \mathrm{U} / 1)\end{array}$ & $282,4 \pm 9,5$ & $304,9 \pm 11,9$ & $308,5 \pm 16,8$ & $308,2 \pm 13,7$ & $288,4 \pm 9,4$ & $290,9 \pm 18,1$ \\
\hline
\end{tabular}

ANOVA de uma via / Teste de Bonferroni (Média \pm EP, $n=14$ homens ). 
globulus Labill até $45 \mathrm{ml}$ ao dia e durante dois meses, foi bem tolerada, não apresentando alterações clínicas, laboratoriais e nem reações adversas significantes. Estes resultados em complementação àqueles obtidos com os ensaios toxicológicos pré-clínicos, sugerem a baixa toxicidade do produto e indicam que esta formulação fitoterápica pode ser utilizada pela população, na dose e via de administração testada. Com estes dados obtidos, pode ser viabilizado o Estudo Clínico, Fase II, deste fitoterápico, de acordo com a Resolução № 251 de 07 de agosto de 1997 do Conselho Nacional de Saúde, em vista do seu registro na Anvisa.

\section{REFERÊNCIAS}

Agra MF, França PF, Barbosa-Filho JM 2007. Synopsis of the plants known as medicinal and poisonous in Northeast of Brazil. Rev Bras Farmacogn 17: 114-140.

Agra MF, Silva KN, Basílio IJLD, França PF, Barbosa-Filho JM 2008. Survey of medicinal plants used in the region Northeast of Brazil. Rev Bras Farmacogn 18: 472508.

Bertolami, MC 2005. Mecanismos de hepatoxicidade. Arq Bras Cardiol 85 (Suppl.): 25-27.

Bertoldi AD, Barros AJD, Hallal PC, Lima RC 2004. Utilização de medicamentos em adultos prevelência e determinantes individuais. Rev Saude Publ 38: 228-
238.

Biavatti M, Marensi V, Leite SN, Reis A 2007. Ethnopharmacognostic survey on botanical compendia for potential cosmeceutic species from Atlantic Forest. Rev Bras Farmacogn 17: 640-653.

Bottini PV, Garlipp CR 2006. Urianálise: comparação entre microscopia óptica e citometria de fluxo. J Bras Patol Med Lab 42: 157-162.

Brandão MGL, Cosenza GP, Moreira RA, Monte-Mor RLM 2006. Medicinal plants and other botanical products from the Brazilian Official Pharmacopoeia. Rev Bras Farmacogn 16: 408-420.

Brasil 1996a. Ministério da Saúde. Secretaria de Vigilância Sanitária. Portaria $N^{\circ} 116$ de 08 de agosto. Norma para Estudo da Toxicidade e da Eficácia de Produtos Fitoterápicos.

Brasil 1996b. Ministério da Saúde. Conselho Nacional de Saúde. Resolução No 196 de 10 de outubro de 1996. Aprova as Diretrizes e Normas Regulamentadoras de Pesquisa Envolvendo Seres Humanos.

Brasil 1997. Ministério da Saúde. Conselho Nacional de Saúde. Resolução N ${ }^{\circ} 251$ de 07 de agosto. Aprova as Normas para a Área de Pesquisa com Novos Fármacos, Medicamentos, Vacinas e Testes Diagnósticos.

Brasil 2004a. Ministério da Saúde. Agência Nacional de Vigilância Sanitária. Resolução RDC nº48, de 16 de março. Dispõe sobre o Registro de medicamentos fitoterápicos.

Tabela 5. Média e desvio padrão dos valores bioquímicos obtidos nas mulheres.

\begin{tabular}{|c|c|c|c|c|c|c|}
\hline Tempo & BASAL & $3^{\circ}$ DIA & $7^{\circ}$ DIA & $3^{\circ}$ SEMANA & $6^{\circ}$ SEMANA & $24 \mathrm{~h} / 8^{\circ}$ SEMANA \\
\hline Exame & $\bar{X} \pm$ E.P. & $\bar{X} \pm$ E.P. & $\bar{X} \pm$ E.P. & $\bar{X} \pm$ E.P. & $\bar{X} \pm$ E.P. & $\bar{X} \pm$ E.P. \\
\hline $\begin{array}{c}\text { Glicemia } \\
(70,0 \text { a } 110,0 \mathrm{mg} / \mathrm{dl})\end{array}$ & $80,4 \pm 1,5$ & $82,9 \pm 1,8$ & $87,6 \pm 3,1$ & $90 \pm 4$ & $82,4 \pm 2,0$ & $84,4 \pm 2,6$ \\
\hline $\begin{array}{l}\text { Colesterol total } \\
\text { (Até } 200 \mathrm{mg} / \mathrm{dl} \text { ) }\end{array}$ & $179,9 \pm 11,8$ & $176,9 \pm 10,7$ & $175 \pm 11,5$ & $173,6 \pm 9,5$ & $187,2 \pm 12,3$ & $184,5 \pm 10,6$ \\
\hline $\begin{array}{l}\text { HDL - Colesterol } \\
\text { (Até } 65 \mathrm{mg} / \mathrm{dl})\end{array}$ & $51,6 \pm 2,6$ & $53,1 \pm 2,3$ & $54,1 \pm 2,5$ & $54,1 \pm 2,7$ & $53,2 \pm 2,4$ & $55,6 \pm 4,3$ \\
\hline $\begin{array}{c}\text { LDL - Colesterol } \\
\text { (Menor que } 130 \mathrm{mg} / \mathrm{dl})\end{array}$ & $113,5 \pm 9,9$ & $109,8 \pm 9,2$ & $106,3 \pm 10,4$ & $105,4 \pm 8,5$ & $118 \pm 10,6$ & $112,6 \pm 9$ \\
\hline $\begin{array}{c}\text { VLDL - Colesterol } \\
\text { (Até 30mg/dl) }\end{array}$ & $13,7 \pm 2,2$ & $15,4 \pm 1,8$ & $14,4 \pm 1,6$ & $13,4 \pm 1,8$ & $15,6 \pm 1,8$ & $15,3 \pm 1,6$ \\
\hline $\begin{array}{c}\text { Uréia } \\
(10 \text { a } 50 \mathrm{mg} / \mathrm{dl})\end{array}$ & $24,3 \pm 1,9$ & $20,4 \pm 1,6$ & $23,9 \pm 2,6$ & $22,2 \pm 2,1$ & $21,9 \pm 1,5$ & $22,0 \pm 3,0$ \\
\hline $\begin{array}{c}\text { Creatinina } \\
(0,4 \text { a } 1,4 \mathrm{mg} / \mathrm{dl})\end{array}$ & $0,5 \pm 0,01$ & $0,5 \pm 0,03$ & $0,5 \pm 0,02$ & $0,5 \pm 0,01$ & $0,6 \pm 0,02$ & $0,4 \pm 0,02$ \\
\hline $\begin{array}{c}\text { CPK } \\
\text { Homem: } \leq 190 \mathrm{U} / 1 \\
\text { Mulher: }<175 \mathrm{U} / 1\end{array}$ & $67,1 \pm 4,3$ & $71,7 \pm 4,7$ & $86,7 \pm 10,9$ & $77,1 \pm 6,1$ & $79 \pm 6,8$ & $73,4 \pm 4,4$ \\
\hline $\begin{array}{l}\text { Ácido Ürico } \\
\text { Homem: } 3 \text { a } 7 \mathrm{mg} / \mathrm{dl} \\
\text { Mulher: } 2 \text { a } 5 \mathrm{mg} / \mathrm{dl}\end{array}$ & $3,4 \pm 0,2$ & $3,8 \pm 0,3$ & $3,4 \pm 0,3$ & $3,1 \pm 0,3$ & $3,2 \pm 0,3$ & $3,4 \pm 0,3$ \\
\hline $\begin{array}{c}\text { Trigliceridios } \\
\text { (Menor que } 200 \mathrm{mg} / \mathrm{dl})\end{array}$ & $74,1 \pm 10,3$ & $81,4 \pm 8,2$ & $73,3 \pm 8,2$ & $71 \pm 8,8$ & $79,7 \pm 9,2$ & $77,9 \pm 7,6$ \\
\hline $\begin{array}{c}\text { Sódio } \\
(132 \mathrm{a} 148 \mathrm{mEq} / \mathrm{l})\end{array}$ & $139,6 \pm 0,5$ & $140,6 \pm 0,4$ & $141 \pm 0,5$ & $140,1 \pm 0,5$ & $140,1 \pm 0,5$ & $140,1 \pm 0,5$ \\
\hline $\begin{array}{c}\text { Potássio } \\
(3,5 \text { a } 5,5 \mathrm{mEq} / \mathrm{l})\end{array}$ & $4,1 \pm 0,07$ & $4,2 \pm 0,06$ & $4,1 \pm 0,07$ & $4,2 \pm 0,08$ & $4,1 \pm 0,06$ & $4,1 \pm 0,08$ \\
\hline
\end{tabular}

ANOVA de uma via / Teste de Bonferroni (Média \pm EP, $n=14$ mulheres). 
Brasil 2004b. Ministério da Saúde. Agência Nacional de Vigilância Sanitária. Resolução RE nº0, de 16 de março. Guia para a realização de estudos de toxicidade Pré-Clínica de Fitoterápicos.

Canigueral S, Vila R 1998. Fitoterapia vademecum de prescripción. 3 ed. Barcelona: Masson.

Carvalho MC, Barca FN, Agnez-Lima LF, Medeiros SR 2003. Evaluation of mutagenic activity in an extract of pepper tree stem bark (Shinus terebinthifolius Raddi). Environ Mol Mutagen 42: 185-191.

Carvalho ACB, Balbino EE, Maciel A, Perfeito JPS 2008. Situação do registro de medicamentos fitoterápicos no
Brasil. Rev Bras Farmacogn 18: 314-319.

Cronquist, A 1981. An Integrated System of Classification of Flowering Plants. Columbia University Press I. New York, 1262p.

Diniz MFFM, Oliveira RAG, Malta JR 1999. Das plantas medicinais aos fitoterápicos - Abordagem multidisciplinar. João Pessoa: PET-Farmácia/CAPES/ UFPB.

Dufour DR 2005. Guias del laboratório para screening, diagnóstico y monitoreo de la lesión hepática. Acta Bioquim Clin Latinoamer 39: 359-376.

Tabela 6. Média e desvio padrão dos valores bioquímicos obtidos nas mulheres (continuação).

\begin{tabular}{|c|c|c|c|c|c|c|}
\hline Tempo & BASAL & $3^{\circ}$ DIA & $7^{\circ}$ DIA & $3^{\circ}$ SEMANA & $6^{\circ}$ SEMANA & $24 \mathrm{~h} / 8^{\circ}$ SEMANA \\
\hline Exame & $\bar{X} \pm$ E.P. & $\bar{X} \pm$ E.P. & $\bar{X} \pm$ E.P. & $\bar{X} \pm$ E.P. & $\bar{X} \pm$ E.P. & $\bar{X} \pm$ E.P. \\
\hline $\begin{array}{c}\text { ALT/TGP } \\
\text { Homem:9 a } 43 \mathrm{U} / 1 \\
\text { Mulher: } 9 \text { a } 36 \mathrm{U} / 1\end{array}$ & $16,9 \pm 2,2$ & $25,8 \pm 2,1$ & $27,4 \pm 3,0$ & $22,5 \pm 1,5$ & $24,0 \pm 2,5$ & $27,2 \pm 3,2$ \\
\hline $\begin{array}{c}\text { AST/TGO } \\
\text { Homem:12 - } 46 \mathrm{U} / 1 \\
\text { Mulher: } 10-31 \mathrm{U} / 1\end{array}$ & $17,2 \pm 1,1$ & $24,1 \pm 1,4$ & $25,2 \pm 2$ & $21,4 \pm 1,3$ & $27,4 \pm 1,6$ & $30,8 \pm 3,2 *$ \\
\hline $\begin{array}{c}\text { Bilirrubina Total } \\
(0,2 \text { a } 1,0 \mathrm{mg} / \mathrm{dl})\end{array}$ & $0,6 \pm 0,07$ & $0,8 \pm 0,09$ & $0,6 \pm 0,05$ & $0,6 \pm 0,06$ & $0,5 \pm 0,05$ & $0,6 \pm 0,05$ \\
\hline $\begin{array}{l}\text { Bilirrubina Direta } \\
(0,05 \text { a } 0,3 \mathrm{mg} / \mathrm{dl})\end{array}$ & $0,1 \pm 0,09$ & $0,2 \pm 0,01$ & $0,2 \pm 0,04$ & $0,1 \pm 0,02$ & $0,1 \pm 0,01$ & $0,1 \pm 0,02$ \\
\hline $\begin{array}{c}\text { Bilirrubina Indireta } \\
\text { (Até } 0,8 \mathrm{mg} / \mathrm{dl} \text { ) }\end{array}$ & $0,5 \pm 0,06$ & $0,6 \pm 0,08$ & $0,5 \pm 0,04$ & $0,5 \pm 0,06$ & $0,4 \pm 0,03$ & $0,5 \pm 0,05$ \\
\hline $\begin{array}{l}\text { Fosfatase Alcalina } \\
\text { (Menor que } 270 \mathrm{U} / \mathrm{l} \text { ) }\end{array}$ & $80,4 \pm 5,1$ & $62,5 \pm 4,7$ & $63,7 \pm 4,2$ & $55,4 \pm 5,0 *$ & $56,9 \pm 5,8 *$ & $53,4 \pm 3,8 *$ \\
\hline $\begin{array}{c}\text { Gama GT } \\
\text { Homem:8 a } 61 \mathrm{U} / 1 \\
\text { Mulher: } 5 \text { a } 36 \mathrm{U} / 1 \\
\end{array}$ & $18,1 \pm 1,7$ & $21,4 \pm 2,0$ & $20,6 \pm 2,3$ & $20,3 \pm 2,7$ & $20,1 \pm 2,7$ & $18,2 \pm 1,9$ \\
\hline $\begin{array}{r}\text { Proteinas Totais } \\
(6,0 \text { a } 8,5 \mathrm{~g} / \mathrm{dl}) \\
\end{array}$ & $7,1 \pm 0,07$ & $7,4 \pm 0,1$ & $7,1 \pm 0,2$ & $7,3 \pm 0,08$ & $7,2 \pm 0,1$ & $7,3 \pm 0,1$ \\
\hline $\begin{array}{c}\text { Albumina } \\
(3,5 \text { a } 5,5 \mathrm{~g} / \mathrm{dl}) \\
\end{array}$ & $4,4 \pm 0,09$ & $4,5 \pm 0,09$ & $4,5 \pm 0,08$ & $4,3 \pm 0,07$ & $4,2 \pm 0,08$ & $4,5 \pm 0,07$ \\
\hline $\begin{array}{c}\text { Globulina } \\
(1,8 \text { a } 3,8 \mathrm{~g} / \mathrm{dl}) \\
\end{array}$ & $2,7 \pm 0,1$ & $2,9 \pm 0,1$ & $2,6 \pm 0,2$ & $2,9 \pm 0,1$ & $2,9 \pm 0,1$ & $2,9 \pm 0,1$ \\
\hline $\begin{array}{c}\text { Amilase Pancreática } \\
(25 \mathrm{a} 125 \mathrm{U} / 1) \\
\end{array}$ & $59,4 \pm 5,9$ & $61,0 \pm 5,6$ & $59,4 \pm 5,6$ & $65,1 \pm 6,2$ & $61,3 \pm 5,6$ & $67,7 \pm 9,5$ \\
\hline $\begin{array}{c}\text { LDH (Lactato Desi- } \\
\text { drogenase } \\
(210 \mathrm{a} 425 \mathrm{U} / \mathrm{l}) \\
\end{array}$ & $265 \pm 8,3$ & $307,4 \pm 19,4$ & $290,8 \pm 10,8$ & $298,2 \pm 13,5$ & $250,4 \pm 13,8$ & $316,6 \pm 19,4$ \\
\hline
\end{tabular}

ANOVA de uma via / Teste de Bonferroni (Média \pm EP, $n=14$ mulheres).

Tabela 7. Reações adversas descritas ao longo do estudo clínico.

\begin{tabular}{lcc}
\hline Efeito & Número & Frequência (\%) \\
\hline Cefaléia & $2 / 28$ & 7,14 \\
Náusea & $1 / 28$ & 3,57 \\
Azia & $2 / 28$ & 7,14 \\
Dor no Estômago & $1 / 28$ & 3,57 \\
Constipação & $2 / 28$ & 7,14 \\
Gosto estranho na boca & $2 / 28$ & 7,14 \\
Diminuição do apetite & $1 / 28$ & 3,57 \\
\hline
\end{tabular}


Lima MR, Luna JS, Santos AF, Andrade MC, Sant'ana AE, Genet JP, Marquez B, Neuville L, Moreau N 2006. Anti-bacterial activity of some Brazilian medicinal plants. J Ethnopharmacol 105: 137-147.

Liu Y, Li S, Wu Y 2003. Advances in the study of Eucalyptus globulus Labill. Zhong Yao Cai 26: 461-473.

Lukhoba CW, Simmonds MS, Paton AJ 2006. Plectranthus: a review of ethnobotanical uses. J Ethnopharmacol 103: 1-24.

Matos FJA 2000. Plantas Medicinais: Guia de seleção e emprego de plantas usadas em fitoterapia no Nordeste do Brasil. 2ed. Fortaleza: Imprensa UniversitáriaUFC.

Medeiros KCP, Monteiro JC, Diniz MFFM, Medeiros IA, Silva BA, Piuvezam MR 2007. Effect of the activity of the Brazilian polyherbal formulation: Eucalyptus globulus Labill, Peltodon radicans Pohl and Schinus terebinthifolius Radd in infl ammatory models. Rev Bras Farmacogn 17: 23-28.

Minker C, Sheridan H, O’Meara J, Visdal LJ, Hook I, Lobstein A, Frankish N 2007. In vivo and in vitro evaluation of anti-inflamatory activity and cytotoxicity of extracts of seven Plectrathus species. Planta Med 73: 54-64.

Moraes MO, Bezerra FAF, Lotufo LC, Pessoa C, Moraes MEA 2004. Avaliação clínica da eficácia e segurança de fitoterápicos no Brasil. Arq Bras Fitomed Cient 1: 3039.

Motta AA, Bianchi PG 2005. Reações adversas a drogas. Rev Med (São Paulo) 84: 10-17.

Oliveira RAG, Lima EO, Vieira WL, Freire KRL, Trajano VN, Lima, IO, Souza EL, Toledo MS, Silva-Filho RN 2006. Estudo da interferência de óleos essenciais sobre a atividade de alguns antibióticos usados na clínica. Rev Bras Farmacogn 16: 77-82.

Oliveira RAG, Lima EO, Souza EL, Vieira WL, Freire KRL, Trajano VN, Lima IO, Silva-Filho RN 2007. Interference of Plectranthus amboinicus (Lour.) Spreng essential oil on the anti-Candida activity of some clinically used antifungals. Rev Bras Farmacogn 17: 186-190.

Santos OJ, Ribas Filho JM, Czeczko NG, Castelo Branco Neto ML, Naufel Júnior C, Ferreira LM, Campos RP, Moreira H, Porcides RD, Dobrowolski S 2006. Evaluation of aroeira (Schinus terebinthifolius Raddi) extract on the healing process of gastroraphy in rats. Acta Cir Bras 21 Suppl 2: 39-45.

Tavares JP, Martins IL, Vieira AS, Lima FAV, Bezerra FAF, Moraes MO, Moraes MEA 2006. Estudo de toxicologia clínica de um fitoterápico a base de associações de plantas, mel e própolis. Rev Bras Farmacogn 16: 350356.

Toscano MGA 2004. Estudos toxicológicos Pré-Clínicos com um Fitoterápico Composto: Eucaliptus globulus, Plectranthus amboinicus e Schinus terebinthifolius. João Pessoa,205p. Tese de Doutorado - Centro de Ciências da Saúde, Laboratório de Tecnologia Farmacêutica, Universidade Federal da Paraíba.
Vigo E, Cepeda A, Gualillo O, Perez-Fernandez R 2004. Invitro anti-inflammatory effect of Eucalyptus globulus and Thymus vulgaris: nitric oxide inhibition in J774A1 murine macrophages. J Pharm Pharmacol 56: 257-263.

Yang XW, Guo QM 2007. Studies on chemical constituents in fruits of Eucalyptus globulus. Zhonqquo Zhong Yao Za Zhi 32: 496-500. 